\title{
Towards optimization by matching of response surfaces: Finding windows of maximal similarity
}

\author{
Veronica Diaz ${ }^{1}$, Mary Acosta ${ }^{1}$, and Mauricio Cabrera-Rios ${ }^{1}$ \\ ${ }^{1}$ University of Puerto Rico Mayaguez
}

May 5, 2020

\begin{abstract}
The ultimate goal of this work is to find a region where the response surface of a function that is not well characterized in terms of optimality resembles one that is well-characterized in such terms to find, at least, a local optimum. The region in the functions' input space where this resemblance occurs, we call a Window of Maximal Similarity (WMS) and is identified by formulating and solving an optimization problem. The method is one of minimization of squared errors and can be used to explore experimental, or simulated data. A series of examples, that include several typical global optimization test functions in literature, are presented in order to demonstrate the method's feasibility and capability for generating a two-dimensional WMS. This tool is a viable element that will serve for the future development of Optimization by Similarity.
\end{abstract}

\section{Hosted file}

TOMRS_ENGR_Template.pdf available at https://authorea.com/users/295829/articles/424701towards-optimization-by-matching-of-response-surfaces-finding-windows-of-maximalsimilarity 